\title{
A Finite Element Method for Solving Helmholtz Type Equations in Waveguides and Other Unbounded Domains*
}

\author{
By Charles I. Goldstein
}

\begin{abstract}
A finite element method is described for solving Helmholtz type boundary value problems in unbounded regions, including those with infinite boundaries. Typical examples include the propagation of acoustic or electromagnetic waves in waveguides. The radiation condition at infinity is based on separation of variables and differs from the classical Sommerfeld radiation condition. It is shown that the problem may be replaced by a boundary value problem on a fixed bounded domain. The behavior of the solution near infinity is incorporated in a nonlocal boundary condition. This problem is given a weak or variational formulation, and the finite element method is then applied. It is proved that optimal error estimates hold.
\end{abstract}

1. Introduction. We shall describe and analyze a numerical procedure based on the finite element method for solving Helmholtz type equations in unbounded regions $\Omega$ with either finite or infinite boundary $\partial \Omega$. When $\partial \Omega$ is bounded (the "exterior problem"), the appropriate radiation condition at infinity that ensures well-posedness of the problem is the classical Sommerfeld radiation condition. There are, however, many important physical problems for which the Sommerfeld radiation condition is not the correct one. This occurs, for example, in connection with the propagation of acoustic or electromagnetic waves in a waveguide [1], as well as wave propagation problems arising in underwater acoustics [2]. In such cases the problem may be shown to be mathematically well-posed using a radiation condition obtained by separation of variables. See [3]-[5] for a proof of this for single waveguides with smooth boundaries. These results were extended in [6] to include more complicated waveguide geometries.

In this paper we describe a method for approximately solving problems of the above type by employing the finite element method on a fixed bounded subset, $\Omega_{B} \subset \Omega$. The behavior of the solution outside of $\Omega_{B}$ is incorporated in a nonlocal boundary condition of the form $\partial u / \partial n=T(u)$ on $\Gamma$, where $\Gamma$ is the outer boundary of $\Omega_{B}, \partial / \partial n$ denotes the outward normal derivative on $\Gamma$, and $T$ is obtained from the radiation condition for the problem. We shall prove that optimal error estimates hold for the finite element method incorporating this nonlocal radiation boundary

\footnotetext{
Received May 27, 1981.

1980 Mathematics Subject Classification. Primary 65N15, 65N30; Secondary 35P25.

*The submitted manuscript has been authored under contract DE-AC02-76CH00016 with the U. S. Department of Energy. Accordingly, the U. S. Government retains a nonexclusive, royalty-free license to publish or reproduce the published form of this contribution, or allow others to do so, for U. S. Government purposes.
} 
condition. We shall carry out the analysis for the case of a single cylindrical waveguide in $\mathrm{N}$-dimensions. In Section 4 we shall indicate other domains for which the method is applicable.

The finite element has been employed by several authors to treat the exterior problem. See, e.g., [7]-[13] and other references cited there, where various formulations of the Sommerfeld radiation condition were employed to incorporate the behavior of the solution near infinity. We shall see in Section 4 that the exterior problem may be considered to be a special case of a conical waveguide. Hence our method yields an alternative approach for solving the exterior problem.

The remainder of this paper may be outlined as follows. In Section 2 we give a precise formulation of our problem for the case of a single cylindrical waveguide in $\mathrm{N}$-dimensions. A variational formulation is given for the solution of this problem. In Section 3 we employ the finite element to approximately solve the problem. Furthermore, we establish optimal error estimates. The main results of the paper are embodied in Theorems 3.1 and 3.2. In Section 4 we discuss more general domains and make some additional observations concerning this method.

2. The Boundary Value Problem in a Cylindrical Waveguide. In this section we consider the Helmholtz equation in a perturbed semi-infinite cylindrical waveguide $\Omega \subset R^{N}$, where $R^{N}$ denotes $N$-dimensional Euclidean space with $N \geqslant 2$. More general domains will be discussed in Section 4 . We begin by introducing our notation. We then establish appropriate outgoing and incoming radiation conditions that ensure the well-posedness of the problem. Finally we shall formulate the radiation condition at infinity in terms of a nonlocal boundary condition on the outer boundary of a fixed bounded subset of $\Omega$ and give a weak or variational formulation of our problem.

We employ the following standard notation for Sobolev spaces of complex-valued functions. Suppose that $M$ is a nonnegative integer, $p \in[1, \infty], B \subset R^{N}$ and $v$ is a complex-valued function defined on $B$. Set

$$
|v|_{W_{p}^{M}(B)}=\max _{|\alpha|=M}\left\|D^{\alpha} v\right\|_{L^{p}(B)}
$$

and

$$
\|v\|_{W_{p}^{M}(B)}=\max _{0 \leqslant m \leqslant M}|v|_{W_{p}^{m}(B)} .
$$

Here $D^{\alpha} v$ denotes the distributional or weak derivative of $v$. Thus \|\|$_{W_{P}^{M}(B)}$ defines a norm and $\left.\right|_{W_{p}^{M}(B)}$ defines a seminorm. The Sobolev spaces are defined via

$$
W_{p}^{M}(B)=\left\{v:\|v\|_{W_{p}^{M}(B)}<\infty\right\},
$$

and the special case $p=2$ is denoted by

$$
H^{M}(B)=W_{2}^{M}(B) \text {. }
$$

Let $H^{M}(B)$ denote the closure of $C_{0}^{\infty}(B)$ in $H^{M}(B)$. For nonintegral $s>0$, we may define $H^{s}(B)$ and \|\|$_{H^{s}(B)}$ in the sense of interpolation theory; see, e.g., [14]. We may also define the space $H^{s}(\Lambda)$ and norm \|\|$_{H^{s}(\Lambda)}$ as in [14], where $\Lambda$ denotes a sufficiently smooth subset of $\partial B$ (the boundary of $B$ ).

We denote an arbitrary point in $R^{N}$ by $x=\left(\tilde{x}, x_{N}\right)$, where $\tilde{x}=\left(x_{1}, \ldots, x_{N-1}\right)$ and $x_{N} \in R^{1}$. We define an unperturbed semi-infinite cylinder $S^{x_{N}^{0}}$ as follows, for some 
$x_{N}^{0} \geqslant 0$. Let $l$ denote a bounded $(N-1)$-dimensional domain. We define $S^{x_{N}^{0}}$ to consist of all points $x=\left(\tilde{x}, x_{N}\right)$ such that $x_{N} \geqslant x_{N}^{0}$ and $\tilde{x} \in l$. (A two-dimensional example of such a domain is a semi-infinite strip: $S^{0}=\left\{\left(x_{1}, x_{2}\right): 0 \leqslant x_{1} \leqslant \pi, 0 \leqslant\right.$ $\left.x_{2}\right\}$.) We now define our waveguide $\Omega$ as follows:

$$
\Omega=\Omega^{0} \cup S^{x_{N}^{0}},
$$

where $\Omega^{0}$ is a bounded set, and we assume that

$$
\Omega \cap\left\{x=\left(\tilde{x}, x_{N}\right): x_{N} \geqslant x_{N}^{0}\right\}=S^{x_{N}^{0}} .
$$

We next consider the following boundary value problem in $\Omega$ :

(2.1) $\left(-\Delta-K^{2}\right) u=f$ in $\Omega, \quad u=0 \quad$ on $\partial \Omega$, and $u$ is "outgoing" at infinity,

where $K$ is real, $f$ and $\partial \Omega$ are smooth, $f$ has bounded support contained in $\Omega-S^{x_{N}^{0}}$, and the "outgoing" radiation condition will be defined shortly. Let $A_{l}$ denote the $(N-1)$-dimensional negative Laplacian acting in $L^{2}(l)$ and associated with the zero Dirichlet boundary condition on $\partial l$. Denote the eigenvalues of $A_{l}$, ordered increasingly, by $\left\{v_{n}\right\}$ and the corresponding orthonormal real-valued eigenfunctions by $\zeta_{n}(\tilde{x})$ for each integer $n \geqslant 1$. (For example, in the case of the semi-infinite strip $S^{0}$ defined above, we see that $\nu_{n}=n^{2}$.)

Suppose that $\nu_{M}<K^{2}<\nu_{M+1}$ for some positive integer $M$. The outgoing radiation condition for $u(x)$ is given by

$$
\left\{\begin{aligned}
& u(x)= \sum_{n=1}^{M} c_{n}(K) e^{i \sqrt{K^{2}-\nu_{n}} x_{N}} \zeta_{n}(\tilde{x})+\sum_{n=M+1}^{\infty} c_{n}(K) e^{-\sqrt{\nu_{n}-K^{2}} x_{N}} \zeta_{n}(\tilde{x}) \\
& \text { and } \\
& \frac{\partial u(x)}{\partial x_{N}}= \sum_{n=1}^{M} i c_{n}(K) \sqrt{K^{2}-v_{n}} e^{i \sqrt{K^{2}-\nu_{n}} x_{N}} \zeta_{n}(\tilde{x}) \\
&-\sum_{n=M+1}^{\infty} c_{n}(K) \sqrt{\nu_{n}-K^{2}} e^{-\sqrt{\nu_{n}-K^{2}} x_{N}} \zeta_{n}(\tilde{x})
\end{aligned}\right.
$$

for each $x_{N} \geqslant x_{N}^{0}$. The incoming radiation condition is similar except that $i \sqrt{K^{2}-\nu_{n}}$ is replaced by $-i \sqrt{K^{2}-\nu_{n}}$ for $1 \leqslant n \leqslant M$.

Remark 2.1. We may generalize problem (2.1) in various ways without essentially modifying our method or results. For example, the Dirichlet boundary condition may be replaced by a Neumann or Robin boundary condition. Furthermore, the differential operator may be perturbed by a variable coefficient second order differential operator with compact support in $\Omega$. Finally, the outgoing radiation condition in (2.1) may be replaced by the incoming radiation condition.

Let $A$ denote the differential operator acting in $L^{2}(\Omega)$ given by $-\Delta$ associated with the zero Dirichlet boundary condition on $\partial \Omega$, and let $\left\{\lambda_{n}\right\}$ denote the set of point eigenvalues of the operator $A$. The set $\left\{\lambda_{n}\right\}$ will be empty in many cases and will be at most countable with no finite accumulation points, [15], even though the continuous spectrum of $A$ consists of the entire semi-infinite interval $\left[\nu_{1}, \infty\right)$. Let $\Lambda$ consist of those real numbers contained in $\left(\nu_{1}, \infty\right)$ other than the sequences $\left\{\nu_{n}\right\}$ and $\left\{\lambda_{n}\right\}$. The following theorem is a consequence of the results in [3] or [4]. 
THEOREM 2.1. Suppose that $K^{2} \in \Lambda$. Then there exists a unique solution $u$ of (2.1). Furthermore, we have

$$
\|u\|_{H^{\prime}(B)} \leqslant C(B, K)\|f\|_{H^{l-2}(B)}
$$

for an arbitrary integer $l \geqslant 2$ and arbitrary subset $B \subset \Omega$, where the constant $C(B, K)$ is independent of $f$.

Note that we employ the notation $C(B, K)$ to indicate that the constant $C(B, K)$ depends on $B$ and $K$. Furthermore, we shall often denote different constants by the same letter when there is no danger of confusion. We next replace problem (2.1) by a boundary value problem on the bounded domain $\Omega_{x_{N}^{\infty}} \subset \Omega$, where

$$
\Omega_{x_{N}^{\infty}}=\Omega \cap\left\{x=\left(\tilde{x}, x_{N}\right): x_{N}<x_{N}^{\infty}\right\} \text { and } x_{N}^{0}<x_{N}^{\infty}<\infty,
$$

with $x_{N}^{\infty}$ fixed throughout the remainder of this paper.

Suppose that $K^{2} \in \Lambda \cap\left(\nu_{M}, \nu_{M+1}\right)$. Set

$$
v_{n}\left(x_{N}^{\prime}\right)=\oint_{\Gamma_{x_{N}^{\prime}}} v(x) \zeta_{n}(\tilde{x}) d \tilde{x}
$$

for arbitrary $v \in L^{2}\left(\Gamma_{x_{N}^{\prime}}\right)$ and $x_{N}^{0} \leqslant x_{N}^{\prime} \leqslant x_{N}^{\infty}$, where

$$
\Gamma_{x_{N}^{\prime}}=\Omega \cap\left\{x=\left(\tilde{x}, x_{N}\right): x_{N}=x_{N}^{\prime}\right\} \text {. }
$$

It follows readily from (2.2) and the orthonormality of the functions $\zeta_{n}(\tilde{x})$ that

$$
\left\{\begin{array}{l}
c_{n}(K)=e^{i \sqrt{K^{2}-\nu_{n}} x_{N}^{\prime}} u_{n}\left(x_{N}^{\prime}\right) \text { for } 1 \leqslant n \leqslant M, \\
\text { and } \\
c_{n}(K)=e^{\sqrt{v_{n}-K^{2}} x_{N}^{\prime}} u_{n}\left(x_{N}^{\prime}\right) \text { for } M<n,
\end{array}\right.
$$

where $x_{N}^{0} \leqslant x_{N}^{\prime} \leqslant x_{N}^{\infty}$. It now follows from (2.2) and (2.5) that the solution $u$ of (2.1) satisfies

$$
\begin{cases}\left(-\Delta-K^{2}\right) u=f & \text { in } \Omega_{x_{N}^{\infty}}, \quad u=0 \text { on } \partial^{\prime} \Omega_{x_{N}^{\infty}} \\ \text { and } & \\ \partial u / \partial x_{N}=T(u) & \text { on } \Gamma_{x_{N}^{\infty}},\end{cases}
$$

where $\partial^{\prime} \Omega_{x_{N}^{\infty}}=\partial \Omega_{x_{N}^{\infty}} \cap \partial \Omega$ and

$$
\begin{aligned}
T(u)(x)= & T_{1}(u)(x)+T_{2}(u)(x) \\
= & i \sum_{n=1}^{M} \sqrt{K^{2}-v_{n}} \zeta_{n}(\tilde{x}) u_{n}\left(x_{N}^{\infty}\right) \\
& -\sum_{n=M+1}^{\infty} \sqrt{v_{n}-K^{2}} \zeta_{n}(\tilde{x}) u_{n}\left(x_{N}^{\infty}\right) \quad \text { for } x \in \Gamma_{x_{N}^{\infty}} .
\end{aligned}
$$

Note that the boundary operators $T, T_{1}$, and $T_{2}$ defined by (2.7) are nonlocal in view of definition (2.4).

Before proceeding further, we define Hilbert spaces $\dot{H}^{s}\left(\Gamma_{x_{N}^{\prime}}\right)$, with $s \in[0,1]$ and $x_{N}^{\prime} \in\left[x_{N}^{0}, x_{N}^{\infty}\right]$, as follows. Let

$$
\dot{H}^{s}\left(\Gamma_{x_{N}^{\prime}}\right)=\left\{v \in L^{2}\left(\Gamma_{x_{N}^{\prime}}\right):\|v\|_{\dot{H}^{s}\left(\Gamma_{x_{N}^{\prime}}\right)}<\infty\right\},
$$


where

$$
\|v\|_{\dot{H}^{s}\left(\Gamma_{x_{N}^{\prime}}\right)}=\left(\sum_{n=1}^{\infty} v_{n}^{s}\left|v_{n}\left(x_{N}^{\prime}\right)\right|^{2}\right)^{1 / 2}
$$

It follows from [16] (Lemma 2.2) that, for $j=0$ and 1 , the spaces $\dot{H}^{j}\left(\Gamma_{x_{N}^{\prime}}\right)$ coincide with the spaces $\dot{H}^{j}\left(\Gamma_{x^{\prime}}\right)$ and the norm on $\dot{H}^{j}\left(\Gamma_{x_{N}^{\prime}}\right)$ is equivalent to the norm on $\stackrel{\circ}{H}^{j}\left(\Gamma_{x_{N}^{\prime}}\right)$. (Note that $\stackrel{\circ}{H}^{0}\left(\Gamma_{x_{N}^{\prime}}\right)=L^{2}\left(\Gamma_{x_{N}^{\prime}}\right)$.) Since the spaces $\dot{H}^{s}\left(\Gamma_{x_{N}^{\prime}}\right)$ define a Hilbert scale connecting the spaces $\dot{H}^{0}\left(\Gamma_{x_{N}^{\prime}}\right)$ and $\dot{H}^{1}\left(\Gamma_{x_{N}^{\prime}}\right)$, it follows from interpolation theory (see, e.g., [14]) that for $s \in[0,1], s \neq \frac{1}{2}$, the spaces $\dot{H}^{s}\left(\Gamma_{x_{N}^{\prime}}\right)$ and $\dot{H}^{s}\left(\Gamma_{x_{N}^{\prime}}\right)$ are identical and the corresponding norms are equivalent. We thus have the following useful estimate:

$$
\begin{aligned}
C_{1}(s)\|v\|_{H^{s}\left(\Gamma_{x_{N}^{\prime}}\right)} & \leqslant\left(\sum_{n=1}^{\infty} v_{n}^{s}\left|v_{n}\left(x_{N}^{\prime}\right)\right|^{2}\right)^{1 / 2} \\
& \leqslant C_{2}(s)\|v\|_{H^{s}\left(\Gamma_{x_{N}^{\prime}}\right)} \text { for each } s \in[0,1], s \neq \frac{1}{2},
\end{aligned}
$$

where $C_{2}(s)$ and $C_{2}(s)$ are independent of $v \in \stackrel{\circ}{H}^{s}\left(\Gamma_{x_{N}^{\prime}}\right)$. Since $\sqrt{\nu_{n}-K^{2}}<\sqrt{\nu_{n}}$ for $n>M$, it follows readily from (2.7), (2.8) and the orthonormality of the functions $\zeta_{n}(\tilde{x})$ that

$$
\|T(v)\|_{L^{2}\left(\Gamma_{\left.x_{n}^{\infty}\right)}\right.} \leqslant C(K)\|v\|_{H^{1}\left(\Gamma_{\left.x_{n}^{\infty}\right)}\right)} \text { for each } v \in \stackrel{\circ}{H}^{1}\left(\Gamma_{x_{N}^{\infty}}\right) .
$$

In order to approximate the solution of (2.6) by the finite element method, we obtain a variational formulation of this problem. We define

$$
H^{E}=\left\{v: v \in H^{1}\left(\Omega_{x_{N}^{\infty}}\right) \cap H^{1}\left(\Gamma_{x_{N}^{\infty}}\right) \text { and } v=0 \text { on } \partial^{\prime} \Omega_{x_{N}^{\infty}}\right\} .
$$

It readily follows, using integration by parts, that the solution $u$ of (2.6) satisfies

$$
a(u, v)=(f, v)=\int_{\Omega_{x_{N}^{\infty}}} f \bar{v} d x, \text { for each } v \in H^{E},
$$

where $a($,$) is defined by$

$$
a(w, v)=\int_{\Omega_{x_{N}^{\infty}}}\left(\nabla w \cdot \nabla \bar{v}-K^{2} w \bar{v}\right) d x-\oint_{\Gamma_{x_{N}^{\infty}}} T(w) \bar{v} d \tilde{x} .
$$

Note that $a(w, v)$ is defined for $w, v \in H^{E}$ since it follows from the Schwarz inequality and (2.9) that

$$
\left|\oint_{\Gamma_{x_{N}^{\infty}}} T(w) \bar{v} d \tilde{x}\right| \leqslant\|T(w)\|_{L^{2}\left(\Gamma_{\left.x_{N}^{\infty}\right)}\right)}\|v\|_{L^{2}\left(\Gamma_{\left.x_{N}^{\infty}\right)}\right.} \leqslant C(K)\|w\|_{H^{1}\left(\Gamma_{\left.x_{N}^{\infty}\right)}\right)}\|v\|_{L^{2}\left(\Gamma_{\left.x_{N}^{\infty}\right)}\right)}<\infty .
$$

Remark 2.2. In order for the numerical method we are describing in this paper to be computationally feasible, it is necessary that the cross section $l$ be simple enough that the eigenvalues $\left\{\nu_{n}\right\}$, eigenfunctions $\left\{\zeta_{n}(\tilde{x})\right\}$, and integrations over $l$ may be easily obtained. This is typically the case in most engineering and physical applications; see, e.g., [17]. Another point worth noting at this time is that the last term in the definition (2.11) of the bilinear form $a($, ) requires the summation of an infinite series, in view of (2.7). We shall see in Theorem 3.2 that suitable error estimates hold even when this series is truncated. 
3. The Finite Element Method. In this section we describe how to approximate the solution of (2.1) using the finite element method. We then establish the well-posedness of the approximate problem and optimal error estimates.

Let $\left\{S^{h}: h \in(0,1]\right\}$ denote a family of finite-dimensional subspaces of $H^{E}$. We shall require the following approximation property of our subspaces $S^{h}$ :

$$
\begin{aligned}
& \inf _{\chi \in S^{h}}\left(\|v-\chi\|_{L^{2}\left(\Omega_{\left.x_{N}^{\infty}\right)}\right.}+h|v-\chi|_{H^{1}\left(\Omega_{x_{N}^{\infty}}\right)}\right. \\
& \left.+h^{1 / 2}\|v-\chi\|_{L^{2}\left(\Gamma_{\left.x_{N}^{\infty}\right)}\right.}+h^{3 / 2}|v-\chi|_{H^{1}\left(\Gamma_{\left.x_{N}^{\infty}\right)}\right)}\right) \leqslant C h^{l^{\prime}}\|v\|_{H^{\prime}\left(\Omega_{x_{N}^{\infty}}\right)}
\end{aligned}
$$

for each $v \in H^{\prime}\left(\Omega_{x_{N}^{\infty}}\right), h \in(0,1]$, and each integer $l^{\prime} \in[2, l]$, where $C$ is independent of $h$ and $v$, and $l$ is an integer greater than one. Estimate (3.1) is satisfied by the usual finite element spaces employed in practice. Typically, such subspaces are constructed by partitioning $\Omega_{x_{N}^{\infty}}$ into simple subsets, $t^{h}$, of diameter $O(h)$ and letting $S^{h}$ consist of sufficiently smooth functions, $v^{h}$, vanishing on $\partial^{\prime} \Omega_{x_{N}^{\infty}}$ and such that $v^{h} \mid t^{h}$ is a polynomial of degree less than $l$.

In view of (2.10), we define our finite element approximation $u^{h}$ to the solution $u$ of $(2.1)$ by the equation

$$
a\left(u^{h}, v^{h}\right)=\left(f, v^{h}\right) \text { for each } v^{h} \in S^{h} .
$$

Choosing a basis for the space $S^{h}$, we obtain from (3.2) a finite system of linear equations to be solved for our desired approximation to the solution on $\Omega_{x_{N}^{\infty}}$. For a comprehensive treatment of the finite element method in connection with elliptic boundary value problems, see [18].

Remark 3.1. In general, it is not always possible to impose the Dirichlet boundary condition on functions in $S^{h}$. (This problem does not arise in the case of a Neumann or Robin boundary condition.) Various methods have been developed for modifying the finite element method so as to circumvent the difficulty associated with the Dirichlet boundary condition (see [18] and the references cited there). We also observe that, for the simple cross sections $l$ employed in practical problems, this boundary condition can usually be imposed on functions in $S^{h}$ everywhere in $\Omega$ except in the bounded set $\Omega_{x_{N}^{0}}$. For the sake of simplicity, we shall employ the finite element method as formulated above.

Our first result establishes the well-posedness of problem (3.2) and optimal error estimates for $u-u^{h}$ when $h$ is sufficiently small.

TheOREM 3.1. Suppose that $u$ satisfies (2.1) with $K^{2} \in \Lambda \cap\left(\nu_{M}, \nu_{M+1}\right)$ and our family of finite element spaces $\left\{S^{h}: h \in(0,1]\right\}$ satisfies assumption (3.1). Then there is an $h_{0}(K) \in(0,1]$ such that there exists a unique solution $u^{h}$ of (3.2) provided $h \in\left(0, h_{0}(K)\right]$. Furthermore, we have

$$
\left\|u-u^{h}\right\|_{L^{2}\left(\Omega_{\left.x_{N}^{\infty}\right)}\right.}+h\left\|u-u^{h}\right\|_{H^{1}\left(\Omega_{\left.x_{N}^{\infty}\right)}\right.} \leqslant C(K) h^{l}\|u\|_{H^{l}\left(\Omega_{\left.x_{N}^{\infty}\right)}\right.}
$$

where $C(K)$ is independent of $h$ and $u$. 
Proof. To begin with, let us assume that there exists a solution $u^{h}$ of (3.2). Set $e^{h}=u-u^{h}$, and apply (2.7) and (2.11) to obtain

$$
a\left(e^{h}, e^{h}\right)=\left|e^{h}\right|_{H^{1}\left(\Omega_{\left.x_{N}^{\infty}\right)}\right)}^{2}-K^{2}\left\|e^{h}\right\|_{L^{2}\left(\Omega_{\left.x_{N}^{\infty}\right)}\right.}^{2}-\oint_{\Gamma_{x_{N}^{\infty}}}\left(T_{1}\left(e^{h}\right)+T_{2}\left(e^{h}\right)\right) \overline{e^{h}} d \tilde{x}
$$

It follows readily from (2.4) (with $v$ replaced by $e^{h}$ ) and (2.7) that

$$
\left\{\begin{array}{l}
\oint_{\Gamma_{x_{N}^{\infty}}} T_{2}\left(e^{h}\right) \overline{e^{h}} d \tilde{x}=-\sum_{n=M+1}^{\infty} \sqrt{\nu_{n}-K^{2}}\left|e_{n}^{h}\left(x_{N}^{\infty}\right)\right|^{2} \\
\text { and } \\
\oint_{\Gamma_{x_{N}^{\infty}}} T_{1}\left(e^{h}\right) \overline{e^{h}} d \tilde{x}=i \sum_{n=1}^{M} \sqrt{K^{2}-\nu_{n}}\left|e_{n}^{h}\left(x_{N}^{\infty}\right)\right|^{2}
\end{array}\right.
$$

Combining (3.3) and (3.4) and equating real parts of the resulting equation, we deduce

$$
\begin{aligned}
\left\|e^{h}\right\|_{H^{1}\left(\Omega_{x_{N}^{\infty}}\right)}^{2}+ & \sum_{n=M+1}^{\infty} \sqrt{\nu_{n}-K^{2}}\left|e_{n}^{h}\left(x_{N}^{\infty}\right)\right|^{2} \\
& =\left(K^{2}+1\right)\left\|e^{h}\right\|_{L^{2}\left(\Omega_{x_{N}^{\infty}}\right)}^{2}+\operatorname{Re} a\left(e^{h}, e^{h}\right)
\end{aligned}
$$

where $\operatorname{Re} a($,$) denotes the real part of a($,$) .$

In order to estimate the last term in (3.5), we apply (2.10), (2.11), (3.1), (3.2), the Schwarz inequality, and the arithmetic-geometric mean inequality to obtain a $\chi \in S^{h}$ such that

$$
\begin{aligned}
\left|a\left(e^{h}, e^{h}\right)\right|= & \left|a\left(e^{h}, u-\chi\right)\right| \\
\leqslant & C h^{l-1}\|u\|_{H^{\prime}\left(\Omega_{x_{N}^{\infty}}\right)}\left(\left|v e^{h}\right|_{H^{1}\left(\Omega_{x_{N}^{\infty}}\right)}+h K^{2}\left\|e^{h}\right\|_{L^{2}\left(\Omega_{x_{N}^{\infty}}\right)}\right) \\
& +\left|\oint_{\Gamma_{x_{N}^{\infty}}} T\left(e^{h}\right)(\overline{u-\chi}) d \tilde{x}\right| \\
\leqslant & C(K, \varepsilon) h^{2(l-1)}\|u\|_{H^{\prime}\left(\Omega_{x_{N}^{\infty}}\right)}^{2}+\varepsilon\left\|e^{h}\right\|_{H^{1}\left(\Omega_{x_{N}^{\infty}}\right)}^{2} \\
& +\left|\oint_{\Gamma_{x_{N}^{\infty}}} T\left(e^{h}\right)(\overline{u-\chi}) d \tilde{x}\right|
\end{aligned}
$$

for arbitrary $\varepsilon \in(0,1]$. It follows from (2.7), (3.1), the trace inequality (see, e.g., [14]), the Schwarz inequality, and the arithmetic-geometric mean inequality that

$$
\begin{gathered}
\left|\oint_{\Gamma_{\Gamma_{N}^{\infty}}} T_{1}\left(e^{h}\right)(\overline{u-\chi}) d \tilde{x}\right| \leqslant\left\|T_{1}\left(e^{h}\right)\right\|_{L^{2}\left(\Gamma_{x_{N}^{\infty}}\right)}\|u-\chi\|_{L^{2}\left(\Gamma_{\left.x_{N}^{\infty}\right)}\right)} \\
\leqslant C(K)\left\|e_{L^{2}\left(\Gamma_{\left.x_{N}^{\infty}\right)}^{h}\right.}\right\| u-\chi \|_{L^{2}\left(\Gamma_{\left.x_{N}^{\infty}\right)}\right)} \\
\leqslant C(K, \varepsilon) h^{2(l-1)}\|u\|_{H^{\prime}\left(\Omega_{x_{N}^{\infty}}\right)}^{2}+\varepsilon\left\|e^{h}\right\|_{H^{1}\left(\Omega_{x_{N}^{\infty}}\right)}^{2}
\end{gathered}
$$


We may now employ (2.4), (2.7), (2.8), (3.1), the Schwarz inequality, and the arithmetic-geometric mean inequality to deduce

$$
\begin{aligned}
& \left|\oint_{\Gamma_{x_{N}^{\infty}}} T_{2}\left(e^{h}\right)(\overline{u-\chi}) d \tilde{x}\right|=\left|\sum_{n=M+1}^{\infty} \sqrt{\nu_{n}-K^{2}} e_{n}^{h}\left(x_{N}^{\infty}\right)(\overline{u-\chi})_{n}\left(x_{N}^{\infty}\right)\right| \\
& \leqslant \varepsilon \sum_{n=M+1}^{\infty} \sqrt{\nu_{n}-K^{2}}\left|e_{n}^{h}\left(x_{N}^{\infty}\right)\right|^{2} \\
& +C(\varepsilon) \sum_{n=M+1}^{\infty} \sqrt{\nu_{n}-K^{2}}(u-\chi)_{n}\left(x_{N}^{\infty}\right) \overline{(u-\chi)_{n}\left(x_{N}^{\infty}\right)} \\
& \leqslant \varepsilon \sum_{n=M+1}^{\infty} \sqrt{\nu_{n}-K^{2}}\left|e_{n}^{h}\left(x_{N}^{\infty}\right)\right|^{2}+C(\varepsilon)\left(\sum_{n=M+1}^{\infty}\left|(u-\chi)_{n}\left(x_{N}^{\infty}\right)\right|^{2}\right)^{1 / 2} \\
& \times\left(\sum_{n=M+1}^{\infty}\left(v_{n}-K^{2}\right)\left|(u-\chi)_{n}\left(x_{N}^{\infty}\right)\right|^{2}\right)^{1 / 2} \\
& \leqslant \varepsilon \sum_{n=M+1}^{\infty} \sqrt{\nu_{n}-K^{2}}\left|e_{n}^{h}\left(x_{N}^{\infty}\right)\right|^{2}+C(\varepsilon)\|u-\chi\|_{L^{2}\left(\Gamma_{\left.x_{N}^{\infty}\right)}\right.}\|u-\chi\|_{H^{1}\left(\Gamma_{\left.x_{N}^{\infty}\right)}\right.} \\
& \leqslant \varepsilon \sum_{n=M+1}^{\infty} \sqrt{\nu_{n}-K^{2}}\left|e_{n}^{h}\left(x_{N}^{\infty}\right)\right|^{2}+C(\varepsilon) h^{2(l-1)}\|u\|_{H^{\prime}\left(\Omega_{\left.x_{N}^{\infty}\right)}^{2}\right)}^{2}
\end{aligned}
$$

Finally, we combine (3.6)-(3.8) to conclude that, for arbitrary $\varepsilon \in(0,1]$, we have

$$
\begin{aligned}
\left|a\left(e^{h}, e^{h}\right)\right| \leqslant & C(K, \varepsilon) h^{2(l-1)}\|u\|_{H^{\prime}\left(\Omega_{\left.x_{N}^{\infty}\right)}\right.}^{2}+2 \varepsilon\left\|e^{h}\right\|_{H^{\prime}\left(\Omega_{x_{N}^{\infty}}\right)}^{2} \\
& +\varepsilon \sum_{n=M+1}^{\infty} \sqrt{\nu_{n}-K^{2}}\left|e_{n}^{h}\left(x_{N}^{\infty}\right)\right|^{2}
\end{aligned}
$$

Set $\varepsilon=\frac{1}{4}$ in (3.9), and then combine this estimate with (3.5) to conclude that

$$
\begin{aligned}
& \frac{1}{2}\left(\left\|e^{h}\right\|_{H^{1}\left(\Omega_{x_{N}^{\infty}}\right)}^{2}+\sum_{n=M+1}^{\infty} \sqrt{\nu_{n}-K^{2}}\left|e_{n}^{h}\left(x_{N}^{\infty}\right)\right|^{2}\right) \\
& \leqslant C(K)\left(h^{2(l-1)}\|u\|_{H^{\prime}\left(\Omega_{\left.x_{N}^{\infty}\right)}\right.}^{2}+\left\|e^{h}\right\|_{L^{2}\left(\Omega_{x_{N}^{\infty}}\right)}^{2}\right)
\end{aligned}
$$

It thus suffices to estimate $\left\|e^{h}\right\|_{L^{2}\left(\Omega_{\left.x_{N}^{\infty}\right)}\right.}$. It follows from the definition of the $L^{2}$ norm that

$$
\begin{aligned}
\left\|e^{h}\right\|_{L^{2}\left(\Omega_{x_{N}^{\infty}}\right)} & =\sup _{\phi \in C_{0}^{\infty}\left(\Omega_{\left.x_{N}^{\infty}\right)}\right.} \frac{\left|\left(e^{h}, \phi\right)\right|}{\|\phi\|_{L^{2}\left(\Omega_{x_{N}^{\infty}}\right)}} \\
& =\sup _{\phi \in C_{0}^{\infty}\left(\Omega_{x_{N}^{\infty}}\right)} \frac{\left|\left(e^{h},\left(-\Delta-K^{2}\right) \Phi\right)\right|}{\|\phi\|_{L^{2}\left(\Omega_{x_{N}^{\infty}}\right)}}
\end{aligned}
$$

where $\Phi$ denotes the solution of (2.1) with $f$ replaced by $\phi$ and the outgoing radiation condition replaced by the incoming radiation condition. It is readily seen, using the 
arguments of Section 2, that $\Phi$ satisfies the following boundary value problem:

$$
\left\{\begin{array}{l}
\left(-\Delta-K^{2}\right) \Phi=\phi \text { in } \Omega_{x_{N}^{\infty}}, \quad \Phi=0 \text { on } \partial^{\prime} \Omega_{x_{N}^{\infty}} \\
\text { and } \\
\frac{\partial \Phi}{\partial x_{N}}=T^{*}(\Phi)=T_{1}^{*}(\Phi)+T_{2}(\Phi) \text { on } \Gamma_{x_{N}^{\infty}},
\end{array}\right.
$$

where

$$
T_{1}^{*}(\Phi)=-i \sum_{n=1}^{M} \sqrt{K^{2}-\nu_{n}} \zeta_{n}(\tilde{x}) \Phi_{n}\left(x_{N}^{\infty}\right)
$$

We now apply integration by parts, (2.7), (2.10), (2.11), (3.2), (3.12), and (3.13) to see that

$$
\begin{aligned}
\left|\left(e^{h},\left(-\Delta-K^{2}\right) \Phi\right)\right| & =\left|\int_{\Omega_{x \infty}}\left(\nabla e^{h} \overline{\nabla \Phi}-K^{2} e^{h} \bar{\Phi}\right) d x-\oint_{\Gamma_{x \otimes ⿱ 丶}} e^{h} \overline{T^{*}(\Phi)} d \tilde{x}\right| \\
& =\left|a\left(e^{h}, \Phi\right)\right|=\left|a\left(e^{h}, \Phi-\chi\right)\right| \text { for each } \chi \in S^{h} .
\end{aligned}
$$

Applying (2.7), (2.8), (2.11), (3.1), the Schwarz inequality, and the argument leading to (3.8), we obtain a $\chi \in S^{h}$ such that

$$
\begin{aligned}
& \left|a\left(e^{h}, \Phi-\chi\right)\right| \\
& \text { (3.15) } \leqslant C h\|\Phi\|_{H^{2}\left(\Omega_{\left.x_{N}^{\infty}\right)}\right)}\left(\left|e^{h}\right|_{H^{1}\left(\Omega_{x_{N}^{\infty}}\right)}+K^{2} h\left\|e^{h}\right\|_{L^{2}\left(\Omega_{\left.x_{N}^{\infty}\right)}\right)}+C(K) h^{1 / 2}\left\|e^{h}\right\|_{L^{2}\left(\Gamma_{x_{N}^{\infty}}\right)}\right. \\
& \left.+\left(\sum_{n=M+1}^{\infty} \sqrt{\nu_{n}-K^{2}}\left|e_{n}^{h}\left(x_{N}^{\infty}\right)\right|^{2}\right)^{1 / 2}\right)
\end{aligned}
$$

Since $\Phi$ is the incoming solution of (2.1) corresponding to data $\phi$, it follows from [3] or [4] that

$$
\|\Phi\|_{H^{2}\left(\Omega_{\left.x_{N}^{\infty}\right)}\right.} \leqslant C\|\Phi\|_{L^{2}\left(\Omega_{\left.x_{N}^{\infty}\right)}\right.} .
$$

Combining (3.11) with (3.14)-(3.16) and the trace inequality, we deduce

$$
\left\|e^{h}\right\|_{L^{2}\left(\Omega_{x_{N}^{\infty}}\right)}^{2} \leqslant C(K) h^{2}\left(\left\|e^{h}\right\|_{H^{1}\left(\Omega_{x_{N}^{\infty}}\right)}^{2}+\sum_{n=M+1}^{\infty} \sqrt{\nu_{n}-K^{2}}\left|e_{n}^{h}\left(x_{N}^{\infty}\right)\right|^{2}\right) .
$$

We may now combine (3.10) and (3.17) to obtain an $h_{0}(K) \in(0,1]$ such that, for $h \in\left(0, h_{0}(K)\right]$, we have

(3.18) $\left\|e^{h}\right\|_{H^{\prime}\left(\Omega_{\left.x_{N}^{\infty}\right)}\right)}^{2}+\sum_{n=M+1}^{\infty} \sqrt{\nu_{n}-K^{2}}\left|e_{n}^{h}\left(x_{N}^{\infty}\right)\right|^{2} \leqslant C(K) h^{2(l-1)}\|u\|_{H^{\prime}\left(\Omega_{\left.x_{N}^{\infty}\right)}\right)}^{2}$.

Finally, we conclude from (3.17) and (3.18) that

$$
\left\|e^{h}\right\|_{L^{2}\left(\Omega_{\left.x_{N}^{\infty}\right)}\right.}+h\left\|e^{h}\right\|_{H^{1}\left(\Omega_{\left.x_{N}^{\infty}\right)}\right.} \leqslant C(K) h^{l}\|u\|_{H^{\prime}\left(\Omega_{\left.x_{N}^{\infty}\right)}\right.} .
$$

We now complete the proof of the theorem as follows. In order to prove that problem (3.2) is well-posed for $h\left(0, h_{0}(K)\right]$, it suffices to prove uniqueness since $S^{h}$ is finite dimensional. Hence, suppose that $u^{h}$ satisfies (3.2) with $f=0$. Since problem 
(2.1) is well-posed by Theorem 2.1, it follows that $u=0$. We thus see from (3.19) that $u^{h}=0$ for $h \in\left(0, h_{0}(K)\right]$, so that (3.2) is well-posed. The remainder of the theorem now follows immediately from (3.19). Q.E.D.

Remark 3.2. It follows from the proof of Theorem 3.1 that $h_{0}(K)$ decreases as $K$ increases. This is typical for numerical methods applied to Helmholtz type equations and is due to the oscillatory nature of the solution.

In view of (2.7) and (2.11), it is necessary to sum an infinite series in order to compute the matrix equations resulting from (3.2). In the remainder of this section, we determine the effect on our error estimates of truncating this infinite series. To this end, we now replace the boundary operator $T$ defined in (2.7) by the operator $T^{J}$ defined as follows for $J \geqslant M+1$ and for each $v \in H^{1}\left(\Gamma_{x_{N}^{\infty}}\right)$ and $\chi \in \Gamma_{x_{N}^{\infty}}$,

$$
\begin{aligned}
T^{J}(v)(x)= & T_{1}(v)(x)+T_{2}^{J} v(x)=T_{1}(v)(x) \\
& -\sum_{n=M+1}^{J-1} \sqrt{v_{n}-K^{2}} \zeta_{n}(\tilde{x}) v_{n}\left(x_{N}^{\infty}\right),
\end{aligned}
$$

where $\sum_{n=M+1}^{J-1}$ is defined to be zero for $J=M+1$.

We define

$$
a^{J}(w, v)=\int_{\Omega_{x_{N}^{\infty}}}\left(\nabla w \cdot \overline{\nabla v}-K^{2} w \bar{v}\right) d x
$$

$$
-\oint_{\Gamma_{x_{N}^{\infty}}} T^{J}(w) \bar{v} d \tilde{x} \quad \text { for each } w, v \in H^{E} .
$$

In our next theorem we show that, for $J$ sufficiently large (independent of $h$ ), the following problem has a unique solution $u_{J}^{h}$ :

$$
a^{J}\left(u_{J}^{h}, v^{h}\right)=\left(f, v^{h}\right), \quad \text { for each } v^{h} \in S^{h} .
$$

THEOREM 3.2. Suppose that $u$ satisfies (2.1) with $K^{2} \in \Lambda \cap\left(\nu_{M}, \nu_{M+1}\right)$ and the family of spaces $\left.\left\{S^{h}: h \in(0,1)\right]\right\}$ satisfies (3.1). Then there exists an $h_{0}(K) \in(0,1]$ and an integer $J_{0}(K) \geqslant M+1$, independent of $h$, such that, for $h \in\left(0, h_{0}(K)\right]$, there exists a unique solution $u_{J}^{h}$ of (3.22) provided $J \geqslant J_{0}(K)$. Furthermore, we have for an arbitrary integer $p \geqslant 1$

$$
\begin{aligned}
& \left\|u-u_{J}^{h}\right\|_{H^{1}\left(\Omega_{\left.x_{N}^{\infty}\right)}\right.}+\left(\sum_{n=M+1}^{J-1} \sqrt{\nu_{n}-K^{2}}\left|\left(u-u_{J}^{h}\right)_{n}\left(x_{N}^{\infty}\right)\right|^{2}\right)^{1 / 2} \\
& \leqslant C(K) h^{l-1}\|u\|_{H^{\prime}\left(\Omega_{\left.x_{N}^{\infty}\right)}\right.}+C e^{-\sqrt{\nu_{J}-K^{2}}\left(x_{N}^{\infty}-x_{N}^{0}\right)} \nu_{J}^{-(p-1) / 2}\|u\|_{H^{p}\left(\Gamma_{x_{N}^{0}}\right)}
\end{aligned}
$$

and

$$
\begin{aligned}
\left\|u-u_{J}^{h}\right\|_{L^{2}\left(\Omega_{\left.x_{N}^{\infty}\right)}\right)} & \left(C(K) h+C(\varepsilon) \nu_{J}^{-\frac{1}{4}+\varepsilon}\right)\left\|u-u_{J}^{h}\right\|_{H^{1}\left(\Omega_{x_{N}^{\infty}}\right)} \\
& +C h\left(\sum_{n=M+1}^{J-1} \sqrt{\nu_{n}-K^{2}}\left|\left(u-u_{J}^{h}\right)_{n}\left(x_{N}^{\infty}\right)\right|^{2}\right)^{1 / 2} \\
& +C e^{-\sqrt{\nu_{J}-K^{2}}\left(x_{N}^{\infty}-x_{N}^{0}\right)} \nu_{J}^{-(p-1) / 2}\|u\|_{H^{p}\left(\Gamma_{x_{N}^{0}}\right)}
\end{aligned}
$$

for $\varepsilon>0$ arbitrarily small. 
Proof. To begin with, we assume that $u_{J}^{h}$ satisfies (3.22) and set $e_{J}^{h}=u-u_{J}^{h}$. As in the proof of Theorem 3.1, it follows from (3.20) and (3.21) that

$$
\begin{aligned}
\left\|e_{J}^{h}\right\|_{H^{1}\left(\Omega_{\left.x_{N}^{\infty}\right)}\right)}^{2}+ & \sum_{n=M+1}^{J-1} \sqrt{\nu_{n}-K^{2}}\left|\left(e_{J}^{h}\right)_{n}\left(x_{N}^{\infty}\right)\right|^{2} \\
& =\left(K^{2}+1\right)\left\|e_{J}^{h}\right\|_{L^{2}\left(\Omega_{x_{N}^{\infty}}\right)}^{2}+\operatorname{Re} a_{J}\left(e_{J}^{h}, e_{J}^{h}\right) .
\end{aligned}
$$

To estimate the last term in (3.25), we observe from (2.7), (2.10), (2.11), and (3.20)-(3.22) that

$$
\begin{array}{r}
a_{J}\left(e_{J}^{h}, e_{J}^{h}\right)=a_{J}\left(e_{J}^{h}, u-\chi\right)-\sum_{n=J}^{\infty} \sqrt{\nu_{n}-K^{2}} u_{n}\left(x_{N}^{\infty}\right) \overline{\left(\chi-u_{J}^{h}\right)_{n}\left(x_{N}^{\infty}\right)} \\
\text { for each } \chi \in S^{h} .
\end{array}
$$

Employing the same argument as in the proof of (3.9), we see that we may choose a $\chi \in S^{h}$ such that, for arbitrary $\varepsilon \in(0,1]$, we have

$$
\begin{aligned}
\left|a_{J}\left(e_{J}^{h}, u-\chi\right)\right| \leqslant & C(K, \varepsilon) h^{2(l-1)}\|u\|_{H^{\prime}\left(\Omega_{x_{N}^{\infty}}\right)}^{2} \\
& +2 \varepsilon\left\|e_{J}^{h}\right\|_{H^{\prime}\left(\Omega_{x_{N}^{\infty}}\right)}^{2}+\varepsilon \sum_{n=M+1}^{J-1} \sqrt{\nu_{n}-K^{2}}\left|\left(e_{J}^{h}\right)_{n}\left(x_{N}^{\infty}\right)\right|^{2} .
\end{aligned}
$$

We next estimate the last term in (3.26). It follows from (2.8), the Schwarz inequality, and the triangle inequality that

$$
\begin{aligned}
& \left|\sum_{n=J}^{\infty} \sqrt{v_{n}-K^{2}} u_{n}\left(x_{N}^{\infty}\right) \overline{\left(\chi-u_{J}^{h}\right)_{n}\left(x_{N}^{\infty}\right)}\right| \\
& \quad \leqslant\left(\sum_{n=J}^{\infty}\left(\nu_{n}-K^{2}\right)\left|u_{n}\left(x_{N}^{\infty}\right)\right|^{2}\right)^{1 / 2}\left(\left\|e_{J}^{h}\right\|_{L^{2}\left(\Gamma_{\left.x_{N}^{\infty}\right)}\right.}+\|u-\chi\|_{L^{2}\left(\Gamma_{x_{N}^{\infty}}\right)}\right) .
\end{aligned}
$$

In view of (2.2) and (2.5), we obtain

$$
\begin{aligned}
u\left(\tilde{x}, x_{N}^{\infty}\right)= & \sum_{m=1}^{M} c_{m}(K) e^{i \sqrt{K^{2}-\nu_{m}} x_{N}^{\infty}} \zeta_{m}(\tilde{x}) \\
& +\sum_{n=M+1}^{\infty} e^{-\sqrt{\nu_{m}-K^{2}}\left(x_{N}^{\infty}-x_{N}^{0}\right)} \zeta_{m}(\tilde{x}) u_{m}\left(x_{N}^{0}\right)
\end{aligned}
$$

It thus follows from the orthonormality of the $\zeta_{m}(\tilde{x})$ that, for $n \geqslant M+1$, we have

$$
u_{n}\left(x_{N}^{\infty}\right)=\oint_{\Gamma_{x_{N}^{\infty}}} u\left(\tilde{x}, x_{N}^{\infty}\right) \zeta_{n}(\tilde{x}) d \tilde{x}=e^{-\sqrt{\nu_{n}-K^{2}}\left(x_{N}^{\infty}-x_{N}^{0}\right)} u_{n}\left(x_{N}^{0}\right)
$$

We now employ (2.8), (3.1), (3.28), (3.30), the trace inequality, and the arithmeticgeometric mean inequality to deduce 


$$
\begin{aligned}
& \left|\sum_{n=J}^{\infty} \sqrt{\nu_{n}-K^{2}} u_{n}\left(x_{N}^{\infty}\right) \overline{\left(\chi-u_{J}^{h}\right)_{n}\left(x_{N}^{\infty}\right)}\right| \\
& \leqslant e^{-\sqrt{\nu_{J}-K^{2}}\left(x_{N}^{\infty}-x_{N}^{0}\right)}\left(\sum_{n=J}^{\infty}\left(\nu_{n}-K^{2}\right)\left|u_{n}\left(x_{N}^{0}\right)\right|^{2}\right)^{1 / 2} \\
& \times\left(\left\|e_{J}^{h}\right\|_{L^{2}\left(\Gamma_{\left.x_{N}^{\infty}\right)}\right.}+\|u-\chi\|_{L^{2}\left(\Gamma_{\left.x_{N}^{\infty}\right)}\right)}\right) \\
& \leqslant C e^{-\sqrt{\nu_{J}-K^{2}}\left(x_{N}^{\infty}-x_{N}^{0}\right)}\left(\|u\|_{H^{\prime}\left(\Gamma_{\left.x_{N}^{0}\right)}^{2}\right.}^{2}+\left\|e_{J}^{h}\right\|_{H^{1}\left(\Omega_{\left.x_{N}^{\infty}\right)}\right.}^{2}+h^{2 l-1}\|u\|_{H^{\prime}\left(\Omega_{x_{N}^{\infty}}\right)}^{2}\right) .
\end{aligned}
$$

Since $\nu_{J}$ increases with $J$, we now choose $\varepsilon=\frac{1}{4}$ in (3.27) and $\nu_{J}$ sufficiently large in (3.31) to conclude from (3.25) and (3.26) that

$$
\begin{aligned}
\frac{1}{4}\left(\left\|e_{J}^{h}\right\|_{H^{1}\left(\Omega_{x_{N}^{\infty}}\right)}^{2}+\sum_{n=M+1}^{J-1} \sqrt{\nu_{n}-K^{2}}\left|\left(e_{J}^{h}\right)_{n}\left(x_{N}^{\infty}\right)\right|^{2}\right) \\
\leqslant\left(K^{2}+1\right)\left\|e_{J}^{h}\right\|_{L^{2}\left(\Omega_{x_{N}^{\infty}}\right)}^{2}+C(K) h^{2(l-1)}\|u\|_{H^{\prime}\left(\Omega_{x_{N}^{\infty}}\right)}^{2} \\
+C e^{-\sqrt{\nu_{J}-K^{2}}\left(x_{N}^{\infty}-x_{N}^{0}\right)}\|u\|_{H^{1}\left(\Gamma_{x_{N}^{0}}\right)}^{2} .
\end{aligned}
$$

We next estimate $\left\|e_{J}^{h}\right\|_{L^{2}\left(\Omega_{x_{N}^{\infty}}\right)}$. It follows as in the proof of Theorem 3.1 that

$$
\left\|e_{H}^{h}\right\|_{L^{2}\left(\Omega_{\left.x_{N}^{\infty}\right)}\right)}=\sup _{\phi \in C_{0}^{\infty}\left(\Omega_{x \mathscr{T}}\right)} \frac{\left|\left(e_{J}^{h}, \phi\right)\right|}{\|\phi\|_{L^{2}\left(\Omega_{\left.x_{N}^{\infty}\right)}\right)}}=\sup _{\phi \in C_{0}^{\infty}\left(\Omega_{\left.x_{N}^{\infty}\right)}\right)} \frac{\left|a\left(e_{J}^{h}, \Phi\right)\right|}{\|\phi\|_{L^{2}\left(\Omega_{\left.x_{N}^{\infty}\right)}\right.}},
$$

where $\phi$ satisfies (3.12). It now follows from (2.7), (2.10), (2.11), and (3.20)-(3.22) that

$$
a\left(e_{J}^{h}, \Phi\right)=a\left(e_{J}^{h}, \Phi-\chi\right)-\sum_{n=J}^{\infty} \sqrt{\nu_{n}-K^{2}}\left(u_{J}^{h}\right)_{n}\left(x_{N}^{\infty}\right) \overline{\chi_{n}\left(x_{N}^{\infty}\right)}
$$

for each $\chi \in S^{h}$. Employing (2.8), (3.16), the argument leading to (3.15), and the trace inequality, we conclude that there exists a $\chi \in S^{h}$ such that

$$
\begin{aligned}
\left|a\left(e_{J}^{h}, \Phi-\chi\right)\right| & \\
\leqslant\|\Phi\|_{H^{2}\left(\Omega_{\left.x_{N}^{\infty}\right)}\right)} \mid & C(K) h\left\|e_{J}^{h}\right\|_{H^{1}\left(\Omega_{\left.x_{N}^{\infty}\right)}\right)} \\
& +\left(\sum_{n=M+1}^{J-1} \sqrt{\nu_{n}-K^{2}}\left|e_{J}^{h}\left(x_{N}^{\infty}\right)\right|^{2}\right)^{1 / 2} \\
& \times\left(\sum_{n=M+1}^{J-1} \sqrt{\nu_{n}-K^{2}}\left|(\Phi-\chi)_{n}\left(x_{N}^{\infty}\right)\right|^{2}\right)^{1 / 2} \\
+ & \left.\left(\sum_{n=J}^{\infty}\left|\left(e_{J}^{h}\right)_{n}\left(x_{N}^{\infty}\right)\right|^{2}\right)^{1 / 2}\left(\sum_{n=J}^{\infty}\left(\nu_{n}-K^{2}\right)\left|(\Phi-\chi)_{n}\left(x_{N}^{\infty}\right)\right|^{2}\right)^{1 / 2}\right) \\
\leqslant\|\phi\|_{L^{2}\left(\Omega_{x_{N}^{\infty}}\right)} & \left(C(K) h\left\|e_{J}^{h}\right\|_{H^{1}\left(\Omega_{\left.x_{N}^{\infty}\right)}\right)}+C h\left(\sum_{n=M+1}^{J-1} \sqrt{\nu_{n}-K^{2}}\left|e_{J}^{h}\left(x_{N}^{\infty}\right)\right|^{2}\right)^{1 / 2}\right.
\end{aligned}
$$

for each $s \in\left[0, \frac{1}{2}\right)$. 
We are thus left with estimating the last term in (3.34). Employing (2.8), the triangle inequality, and the Schwarz inequality, we obtain

$$
\begin{aligned}
\mid \sum_{n=J}^{\infty} \sqrt{\nu_{n}-K^{2}} & \left(u_{J}^{h}\right)_{n}\left(x_{N}^{\infty}\right) \overline{\chi_{n}\left(x_{N}^{\infty}\right)} \mid \\
\leqslant & \left(\sum_{n=J}^{\infty}\left(\nu_{n}-K^{2}\right)\left|u_{n}\left(x_{N}^{\infty}\right)\right|^{2}\right)^{1 / 2}\left(\|\Phi-\chi\|_{L^{2}\left(\Gamma_{\left.x_{N}^{\infty}\right)}\right.}+\|\Phi\|_{L^{2}\left(\Gamma_{\left.x_{N}^{\infty}\right)}\right)}\right) \\
& +\left(\sum_{n=J}^{\infty}\left|\left(e_{J}^{h}\right)_{n}\left(x_{N}^{\infty}\right)\right|^{2}\right)^{1 / 2}\left(\|\Phi-\chi\|_{H^{1}\left(\Gamma_{\left.x_{N}^{\infty}\right)}\right.}+\|\Phi\|_{H^{1}\left(\Gamma_{\left.x_{N}^{\infty}\right)}\right)}\right) .
\end{aligned}
$$

We now combine (2.8), (3.1), (3.16), (3.30), and the trace inequality to deduce

$$
\begin{aligned}
&\left|\sum_{n=J}^{\infty} \sqrt{\nu_{n}-K^{2}}\left(u_{J}^{h}\right)_{n}\left(x_{N}^{\infty}\right) \overline{\chi_{n}\left(x_{N}^{\infty}\right)}\right| \\
& \leqslant C e^{-\sqrt{\nu_{J}-K^{2}}\left(x_{N}^{\infty}-x_{N}^{0}\right)}\|u\|_{H^{1}\left(\Gamma_{\left.x_{N}^{0}\right)}\right.}\|\phi\|_{L^{2}\left(\Omega_{\left.x_{N}^{\infty}\right)}\right)} \\
&+C(s) \nu_{J}^{-s / 2}\left\|e_{J}^{h}\right\|_{H^{s}\left(\Gamma_{\left.x_{N}^{\infty}\right)}\right)}\|\phi\|_{L^{2}\left(\Omega_{x_{N}^{\infty}}\right)}
\end{aligned}
$$

for each $s \in\left[0, \frac{1}{2}\right)$. Finally, we apply (2.8), (3.32)-(3.36), and the trace inequality and choose $h$ sufficiently small in (3.35) and $J$ sufficiently large (independent of $h$ ) in (3.36) to conclude that

$$
\begin{aligned}
& \left\|e_{J}^{h}\right\|_{H^{1}\left(\Omega_{\left.x_{N}^{\infty}\right)}\right)}+\left(\sum_{n=M+1}^{J-1} \sqrt{\nu_{n}-K^{2}}\left|\left(e_{J}^{h}\right)_{n}\left(x_{N}^{\infty}\right)\right|^{2}\right)^{1 / 2} \\
& \quad \leqslant C(K) h^{l-1}\|u\|_{H^{\prime}\left(\Omega_{\left.x_{N}^{\infty}\right)}\right.}+C e^{-\sqrt{\nu_{J}-K^{2}}\left(x_{N}^{\infty}-x_{N}^{0}\right)}\|u\|_{H^{1}\left(\Gamma_{x_{N}^{0}}\right)}
\end{aligned}
$$

and

$$
\begin{aligned}
\left\|e_{J}^{h}\right\|_{L^{2}\left(\Omega_{\left.x_{N}^{\infty}\right)}\right)} \leqslant & \left(C(K) h+C(s) \nu_{J}^{-s / 2}\right)\left\|e_{J}^{h}\right\|_{H^{1}\left(\Omega_{\left.x_{N}^{\infty}\right)}\right.} \\
& +C h\left(\sum_{n=M+1}^{J-1} \sqrt{\nu_{n}-K^{2}}\left|\left(e_{J}^{h}\right)_{n}\left(x_{N}^{\infty}\right)\right|^{2}\right)^{1 / 2} \\
& +C e^{-\sqrt{\nu_{J}-K^{2}}\left(x_{N}^{\infty}-x_{N}^{0}\right)}\|u\|_{H^{1}\left(\Gamma_{\left.x_{N}^{0}\right)}\right.}
\end{aligned}
$$

for each $s \in\left[0, \frac{1}{2}\right)$.

The well-posedness of problem (3.22) for $h$ sufficiently small and $J$ sufficiently large now follows from (3.37) and the finite dimensionality of $S^{h}$ in the same manner as the analogous result in Theorem 3.1. Furthermore, it follows from (3.37) and (3.38) that the theorem is proved with $p=1$. In order to prove (3.23) and (3.24) for $p>1$, we use the following observation due to J. Pasciak. Since $\operatorname{supp}(f) \subset \Omega_{x_{N}^{0}}$, we readily see, using (2.1) and (2.2), that $u \in C^{\infty}\left(\Gamma_{x_{N}^{0}}\right)$ and $A_{l}^{q} u=0$ on $\partial \Gamma_{x_{N}^{0}}$ for each integer $q \geqslant 0$, where the operator $A_{l}$ was defined in Section 2 as the $(N-1)$ dimensional negative Laplacian acting in $L^{2}(l)$. Hence it follows from [16] that

$$
C_{1}(p)\|u\|_{H^{p}\left(\Gamma_{\left.x_{N}^{0}\right)}\right)} \leqslant\left(\sum_{n=1}^{\infty} \nu_{n}^{p}\left|u_{n}\left(x_{N}^{0}\right)\right|^{2}\right)^{1 / 2} \leqslant C_{2}(p)\|u\|_{H^{p}\left(\Gamma_{\left.x_{N}^{0}\right)}\right)}
$$


with $C_{1}(p) \leqslant C_{2}(p)<\infty$. We may now combine (3.39) with the proof of (3.31) and (3.36) to obtain (3.23) and (3.24). Q.E.D.

4. Additional Comments. In this section we indicate how the theory presented in Sections 2 and 3 may be extended in various ways. We intend to investigate many of these extensions in detail in future publications. To begin with, we observe from (2.2) that only the first $M$ modes of the solution $u$ of (2.1) are propagating out to infinity, while all of the remaining terms in (2.2) are exponentially decaying. (This is in contrast to some of the other waveguides we shall discuss below, such as conical waveguides, for which there are no exponentially decaying modes.) Hence it seems reasonable that a good approximation to the solution of (2.1) may be obtained by employing a boundary operator on $\Gamma_{x_{N}^{\infty}}$ that is exact for solutions containing only the first $M$ terms in series (2.2).

Such a boundary operator is given by the operator $T_{1}$ defined in (2.7) for $K^{2} \in \Lambda \cap\left(\nu_{M}, \nu_{M+1}\right)$ by

$$
T_{1} u(x)=i \sum_{n=1}^{M} \sqrt{K^{2}-\nu_{n}} \zeta_{n}(\tilde{x}) \oint_{\Gamma_{x_{N}^{\infty}}} u\left(\tilde{x}^{\prime}, x_{N}^{\infty}\right) \zeta_{n}\left(\tilde{x}^{\prime}\right) d \tilde{x}^{\prime} .
$$

Another boundary operator that is exact for the first $M$ terms of series (2.2) is given by

$$
B_{M} u(x)=\prod_{n=1}^{M}\left(\frac{\partial}{\partial x_{N}}-i \sqrt{K^{2}-\nu_{n}}\right) u(x)
$$

The well-posedness of these approximate boundary value problems, as well as a finite element analysis of these problems and the proof of a suitable error estimate for the difference between the solution of the exact and approximate problems, will be considered elsewhere. Note that $B_{M}$ is a local operator, and hence the resulting matrix equations will be sparse. Since $B_{M}$ is a differential operator of order $M$, the smoothness requirements of our finite element subspaces on the outer boundary must be increased as $M$ increases. A sequence of local, higher order differential boundary operators for approximately solving the exterior problem was investigated in [7].

There are various other problems for which all but a finite number of modes are exponentially decaying. This is true, for example, in the case of a compound cylindrical waveguide consisting of a finite union of semi-infinite cylinders, as well as wave propagation problems in underwater acoustics (see [2]). There are, however, a great many domains for which none of the modes are exponentially decaying. We shall illustrate this for the case of a perturbed two-dimensional cone.

Let

$$
\Omega=\Omega^{0} \cup S^{r_{0}},
$$

where $r_{0} \geqslant 0, \Omega^{0}$ is a smooth, bounded set, $S^{r_{0}}=\left\{x=(r, \theta): r_{0} \leqslant r, 0 \leqslant \theta \leqslant \pi \alpha\right\}$, $(r, \theta)$ denotes the polar coordinates of a point $x$ in $R^{2}$, and $0<\alpha \leqslant 2$. We again consider problem (2.1) with respect to this domain. It was proved in [5] that this 
problem is well-posed for any real $K \neq 0$ with the outgoing radiation condition defined by

$$
\left\{\begin{array}{l}
u(x)=\sum_{n=1}^{\infty} c_{n}(K) H_{n / \alpha}^{(1)}(K r) \sin \frac{n}{\alpha} \theta \\
\text { and } \\
\frac{\partial u}{\partial r}(x)=\sum_{n=1}^{\infty} c_{n}(K) \frac{d}{d r} H_{n / \alpha}^{(1)}(K r) \sin \frac{n}{\alpha} \theta
\end{array}\right.
$$

where $H_{n / \alpha}^{(1)}(K r)$ denotes the Hankel function of the first kind. The incoming radiation condition is defined similarly with $H_{n / \alpha}^{(1)}$ replaced by $H_{n / \alpha}^{(2)}$ (the Hankel function of the second kind). Proceeding as in Section 2, we obtain the following boundary value problem:

$$
\left(-\Delta-K^{2}\right) u=f \quad \text { in } \Omega_{r_{\infty}}, \quad u=0 \quad \text { on } \partial^{\prime} \Omega_{r_{\infty}} \text { and } \frac{\partial u}{\partial r}=T(u) \text { on } \Gamma_{r_{\infty}},
$$

where $r_{\infty}>r_{0}, \Omega_{r_{\infty}}=\Omega \cap\left\{x=(r, \theta): r<r_{\infty}\right\}, \quad \partial^{\prime} \Omega_{r_{\infty}}=\partial \Omega_{r_{\infty}} \cap \partial \Omega, \Gamma_{r_{\infty}}=\Omega \cap$ $\left\{x=(r, \theta): r=r_{\infty}\right\}$ and

$$
T(u)\left(r_{\infty}, \theta\right)=(\pi \alpha)^{-1} \sum_{n=1}^{\infty} \frac{(d / d r) H_{n / \alpha}^{(1)}\left(K r_{\infty}\right)}{H_{n / \alpha}^{(1)}\left(K r_{\infty}\right)} u_{n}\left(r_{\infty}\right) \sin \frac{n}{\alpha} \theta
$$

with

$$
u_{n}\left(r_{\infty}\right)=\int_{0}^{\pi \alpha} u\left(r_{\infty}, \theta\right) \sin \frac{n}{\alpha} \theta d \theta
$$

Introducing a family of finite element spaces $S^{h}$ as in Section 3, we obtain in an analogous fashion the following equation for the finite element approximation $u^{h} \in S^{h}$ of the solution $u$ :

$$
\begin{aligned}
a\left(u^{h}, v^{h}\right)= & \int_{\Omega_{r_{\infty}}}\left(\nabla u^{h} \cdot \overline{\nabla v^{h}}-K^{2} u^{h} \overline{v^{h}}\right) d x \\
& -\oint_{\Gamma_{r_{\infty}}} T\left(u^{h}\right) \overline{v^{h}} d \theta=\left(f, v^{h}\right) \quad \text { for each } v^{h} \in S^{h}
\end{aligned}
$$

It is readily seen, using (4.3) and (4.4), that

$$
\oint_{\Gamma_{r_{\infty}}} T\left(u^{h}\right) \overline{v^{h}} d \theta=(\pi \alpha)^{-1} \sum_{n=1}^{\infty} \frac{(d / d r) H_{n / \alpha}^{(1)}\left(K r_{\infty}\right)}{H_{n / \alpha}^{(1)}\left(K r_{\infty}\right)} u_{n}^{h}\left(r_{\infty}\right) \overline{v_{n}^{h}\left(r_{\infty}\right)}
$$

As in Section 3, it may be seen that it suffices to take a finite number of terms in the infinite series (4.5). In this case, however, the higher order terms in (4.2) are not exponentially decaying, so that a larger number of terms will be required in general than for cases in which all but a finite number of terms decay exponentially.

Observe that the exterior problem in $R^{2}$ is a special case of the present problem with $\alpha=2$. Hence our method yields an alternative approach to solving the exterior scattering problem. The nonlocal boundary condition on $\Gamma_{r_{\infty}}$ is obtained without having to invert an integral operator. In addition, variable coefficients may be treated since the Green's function need not be known. 
The well-posedness results of [3]-[5] were extended in [6] to a large class of waveguides in three dimensions, including compound waveguides consisting of a finite union of single waveguides of (possibly) different geometries. The results in [6] also included waveguides with discontinuities. It may be seen that the finite element method proposed here can be readily extended to these more general geometries (providing, of course, that appropriate modifications are made when discontinuities are present).

Acknowledgment. The author wishes to express his gratitude to Dr. J. Pasciak for several useful discussions and comments concerning this work, and in particular for his suggestion leading to a sharpening of the estimates in Theorem 3.2.

Applied Mathematics Department Brookhaven National Laboratory Upton, New York 11973

1. C. I. GoldSTEIN, "Scattering theory in waveguides," Scattering Theory in Mathematical Physics (J. A. LaVita and J. P. Marchand, editors), Reidel, Dordrecht, 1974, pp. 35-52.

2. G. FIX \& S. P. MARIN, "Variational methods for underwater acoustic problems," J. Comput. Phys. v. 28,1978, pp. $253-270$.

3. D. M. EIDus, “The principle of limiting absorption," Amer. Math. Soc. Transl. (2), v. 47, 1965, pp. 157-191.

4. C. I. GolDSTEIN, "Eigenfunction expansions associated with the Laplacian for certain domains with infinite boundaries. I," Trans. Amer. Math. Soc., v. 135, 1969, pp. 1-31.

5. C. I. GoldSTEIN, "Eigenfunction expansions associated with the Laplacian for certain domains with infinite boundaries. III," Trans. Amer. Math. Soc., v. 143, 1969, pp. 283-301.

6. J. C. Guillot \& C. H. Wilcox, "Steady state wave propagation in simple and compound acoustic waveguides," Math. Z., v. 160, 1978, pp. 89-102.

7. A. Bayliss, M. GunZBerger \& E. TURKel, Boundary Conditions for the Numerical Solution of Elliptic Equations in Exterior Regions, ICASE Report 80-1, 1979.

8. C. I. GoldsteIn, "The finite element method with nonuniform mesh sizes for unbounded domains," Math. Comp., v. 36, 1981, pp. 387-404.

9. C. I. Goldstein, "The finite element method with nonuniform mesh sizes applied to the exterior Helmholtz problem," Numer. Math., v. 38, 1981, pp. 61-82.

10. F. BRezzi, C. Johnson \& J. C. NedeleC, On the coupling of boundary integral and finite element methods, Ecole Polytechnique Report No. 39, 1978.

11. S. MARIN, A Finite Element Method for Problems Involving the Helmholtz Equation in Two Dimensional Exterior Regions, Thesis, Carnegie-Mellon University, Pittsburgh, Pa., 1978.

12. A. K. Aziz \& R. B. KellogG, "Finite element analysis of a scattering problem," Math. Comp., v. 37, 1981, pp. 261-272.

13. J. H. Bramble \& J. E. Pasciak, A New Computational Approach for the Linearized Scalar Potential Formulation of the Magnetostatic Field Problem, BNL AMD Report No. 872. (Preprint.)

14. J. L. Lions \& E. Magenes, Non-Homogeneous Boundary Value Problems and Applications, Vol. I (English), Springer-Verlag, Berlin and New York, 1972.

15. D. S. Jones, "The eigenvalues of $\Delta u+\lambda u=0$ when the boundary conditions are given on semi-infinite domains," Proc. Cambridge Philos. Soc., v. 49, 1953, pp. 668-684.

16. J. H. BRAmble \& V. ThomEe, "Discrete time Galerkin methods for a parabolic boundary value problem," Ann. Mat. Pura Appl., v. 101, 1974, pp. 115-152.

17. D. S. Jones, The Theory of Electromagnetism, Pergamon Press, New York, 1964.

18. P. G. Ciarlet, The Finite Element Method for Elliptic Problems, North-Holland, Amsterdam, 1978. 